\title{
Evidence for the spread of the alien species Aedes koreicus in the Lombardy region, Italy
}

\author{
Agata Negri ${ }^{1,4 \dagger}$, Irene Arnoldi ${ }^{2,3,4 \dagger}$, Matteo Brilli ${ }^{1}$, Claudio Bandi ${ }^{1,4}$, Paolo Gabrieli ${ }^{1,4^{*}}$ and Sara Epis ${ }^{1,4^{*}}$ (D)
}

\begin{abstract}
Background: Aedes koreicus is a mosquito species characterized by marked anthropophilic behavior, and a potential vector of nematodes and viruses. It is native to East Asia, but its presence has recently been reported in many regions of Europe. In Italy, these mosquitoes had been detected in the northeast since 2011 and are now spreading towards the southwest of the country.

Methods: In 2020, during a surveillance program for invasive mosquito species in the district of Bergamo (Lombardy Region, Italy), about 6000 mosquito larvae were collected. Emerged adults were assigned to mosquito species according to morphological analyses, followed by amplification and sequencing of genetic markers (COI, ND4, ITS2 and D2).

Results: According to the morphological and genetic data, about 50 individuals belonged to the species Ae. koreicus. Conclusion: We report the presence of Ae. koreicus in the district of Bergamo, which confirms the spread of this species in the north of Italy and raises concerns about its possible role as a vector of diseases in the Alpine area.
\end{abstract}

Keywords: Alien species, Aedes koreicus, Invasive mosquitoes, Morphological and molecular identification

In recent decades, the spread of alien mosquito species, as potential vectors of pathogens of public health importance, has raised serious concern in Europe $[1,2]$. In addition to the well-known tiger mosquito Aedes albopictus, another species of this genus, Ae. koreicus, is spreading in Europe $[2,3]$. This species is native to East Asia (Japan, Korea, China and Russia) [4] and was reported in Europe for the first time in Belgium in 2008, in the industrial zone of Maasmechelen [5]. Subsequently, individuals from this species have been repeatedly collected, starting from 2011 in Belluno and in the northeast mountainous region of Italy [6-10], and then in 2012 and 2013 at the Swiss-Italian border (Chiasso and Como) [11], in 2013 in a village in northeastern Slovenia [12], in 2015 in

\footnotetext{
*Correspondence: paolo.gabrieli@unimi.it; sara.epis@unimi.it

${ }^{\dagger}$ Agata Negri and Irene Arnoldi contributed equally to this work. To whom correspondence may be addressed.E-mail: sara.epis@unimi.it or paolo. gabrieli@unimi.it

${ }^{1}$ Department of Biosciences and Pediatric Clinical Research Center "Romeo Ed Enrica Invernizzi", University of Milan, 20133 Milan, Italy

Full list of author information is available at the end of the article
}

southern Germany [13] and Genoa (Italy) [14], in 2016 in central Germany [15] and Pécs (Hungary) [16], and in 2018 in East Tyrol in Austria [17].

Here we report the detection of a novel population of Ae. koreicus in Italy, in the Bergamo district (in the Lombardy region). This novel record highlights that this species is likely expanding in Lombardy, especially in mountain areas. Identification of the collected mosquitoes was achieved by morphological observations and molecular analysis of three genetic markers, which were then also used to reconstruct phylogenetic trees. Our record further emphasizes the urgent need for coordinated efforts for the monitoring of invasive mosquitoes in Italy, to prevent the spread of vectors of emerging pathogens.

In a surveillance program for invasive mosquito species in the Bergamo district $\left(45^{\circ} 43^{\prime} 02.3^{\prime \prime} \mathrm{N} 9^{\circ} 50^{\prime} 38.0^{\prime \prime}\right.$ E, $370 \mathrm{~m}$ above sea level, Lombardy Region, Italy) (Additional file 1: Figure S1), about 6000 larvae and hundreds of mosquito eggs were collected from a pond and several artificial tanks; we collected larvae and eggs during original author(s) and the source, provide a link to the Creative Commons licence, and indicate if changes were made. The images or other third party material in this article are included in the article's Creative Commons licence, unless indicated otherwise in a credit line to the material. If material is not included in the article's Creative Commons licence and your intended use is not permitted by statutory regulation or exceeds the permitted use, you will need to obtain permission directly from the copyright holder. To view a copy of this licence, visit http://creativecommons.org/licenses/by/4.0/. The Creative Commons Public Domain Dedication waiver (http://creativeco mmons.org/publicdomain/zero/1.0/) applies to the data made available in this article, unless otherwise stated in a credit line to the data. 
four catches, between July and September 2020 (end of July, mid-August, and the beginning and end of September). Samples were then reared in the insectarium under standard conditions $\left(26{ }^{\circ} \mathrm{C}, 80 \%\right.$ humidity). After each harvest, the larvae were separated into three groups, according to the observed morphological differences; About 4800 larvae were identified as belonging to the genus Culex, based on the recognition of the peculiar traits of this genus at the larval instar. Some of the remaining larvae were observed and morphologically characterized under a stereomicroscope before pupation; most of them emerged as adults and were observed for further identification. Based on the morphological identification keys described in Tanaka et al. [4], Versteirt et al. [5] and Pfitzner et al. [15], about 50 larvae and adult mosquitoes were identified as Ae. koreicus. The remaining mosquitoes were identified as belonging to the genus Culiseta.
As reported in Fig. 1, the head of Ae. koreicus larvae shows the four head hairs (cranial setae) lining the frontal clipeal edge and about 18 evenly spaced teeth on siphonal pecten. Moreover, adult mosquitoes of Ae. koreicus show a pale basal band at the hind tarsomeres IV and the typical scutal pattern. The base of the posterior femur is completely pale, and a subspiracular patch of pale scales is usually present. These features distinguish Ae. koreicus from the closely related invasive mosquito Ae. japonicus japonicus, which instead usually presents dark hind tarsomeres IV and a dark subbasal band on the posterior femur. Moreover, Ae. j. japonicus mosquitoes lack the subspiracular patch [15]. In recent years, two main populations of Ae. koreicus have been identified as invasive mosquitoes in Europe: the Korean mainland population and the South Korean volcanic island Jeju population [4, 5]. The two populations can be partially distinguished by morphological analysis, since the Korean mainland population is characterized by a usually dark hind tarsomere

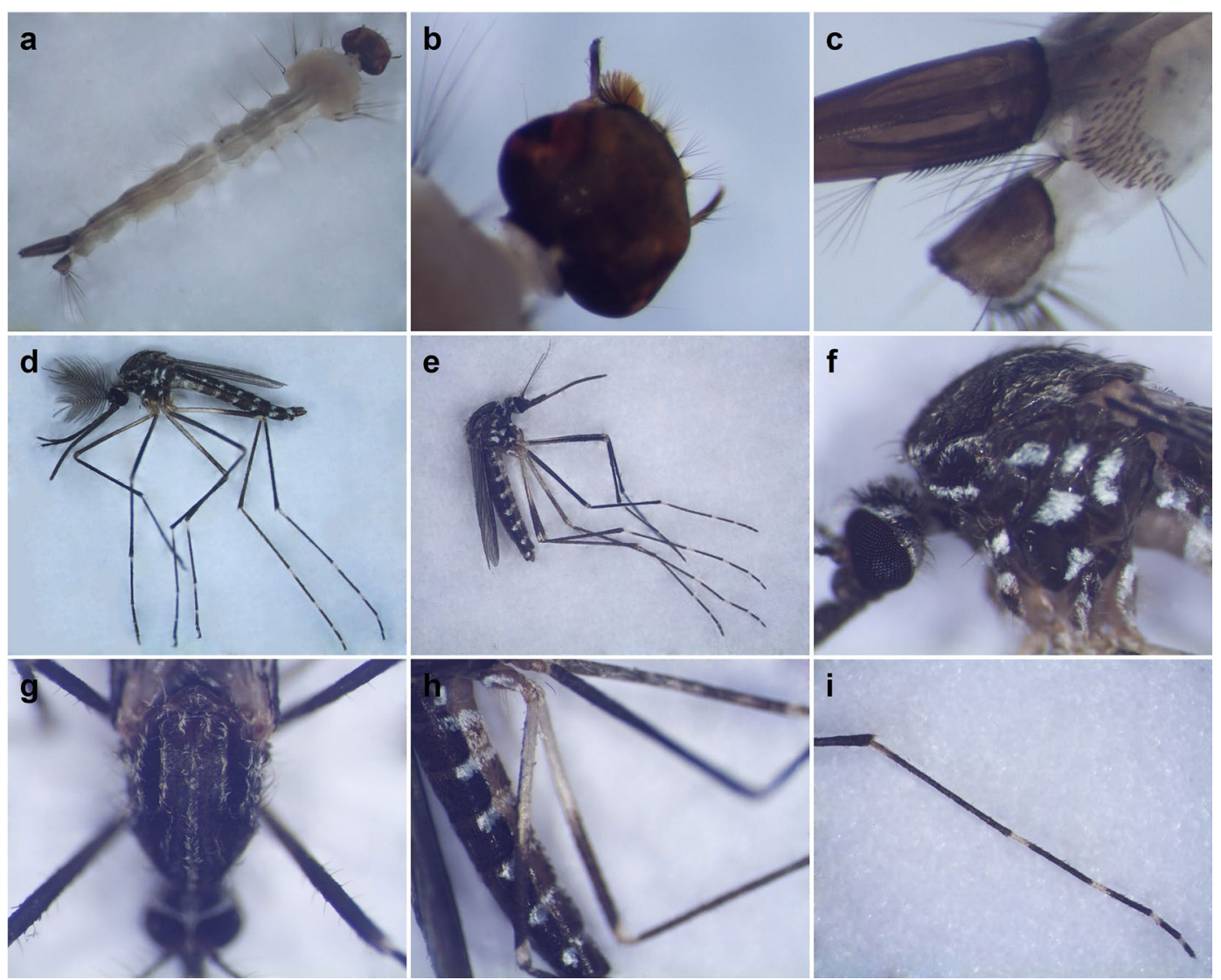

Fig. 1 Morphological features of Aedes koreicus larvae $(\mathbf{a}-\mathbf{c})$ and adult mosquitoes $(\mathbf{d}-\mathbf{i})$. The fourth-instar larva (a) carries setae with multiple branches in the frontal region of the head (b), while about 18 evenly spaced teeth are located on the siphonal pecten (c). Male (d) and female (e) adults show typical sexual dimorphism. The subspiracular area has a distinct patch of pale scales (f); the dark mesonotum harbors five defined lines forming a peculiar pattern with yellowish-brown or golden-yellow scales (g); the posterior femurs are characterized by a completely pale base (h), and the hind tarsomere IV shows the typical pale basal band (i) 
$\mathrm{V}$ [4]. On the contrary, the Jeju population presents, in the same body part, a pale basal band, a characteristic also observed in Ae. koreicus mosquitoes collected and maintained in our insectarium. Thus, we could attribute the individuals of Ae. koreicus collected in Lombardy to the Jeju morphological variant, which was previously reported in Belgium [5] and in the Veneto region in Italy [6].

To confirm the morphological identification, molecular analyses were performed on adult female mosquitoes. Methods for molecular identification of mosquito species, such as the one developed by Cameron et al. (2010) [18], are useful for the identification of field-collected specimens. Indeed, morphological identification of Ae. koreicus can be misleading because of peculiar features partially overlapping with those of Ae. japonicus subspecies. Moreover, samples can be damaged: from our experience, some morphological characters, such as the scales forming the design of the mesonotum, can be lost if mosquitoes are stored for a long time, making the identification difficult. In this study, following polymerase chain reaction (PCR)-based identification as described in Cameron et al. [18], the sequencing of four loci (COI, D2, ND4 and ITS) allowed us to further confirm the identification of the collected samples and to reconstruct the phylogenetic relationship between Ae. koreicus and other mosquito species. DNA was extracted from whole bodies of individual mosquitoes (Monarch ${ }^{\circledR}$ Genomic DNA Purification Kit, New England Biolabs). A first molecular analysis was performed following Cameron et al. [18]: the consistent presence of a single-nucleotide polymorphism (SNP) in the nicotinamide adenine dinucleotide dehydrogenase subunit 4 (ND4) gene of Ae. koreicus has enabled the development of a PCR tool for rapid identification of this species. This PCR protocol uses the Ae. koreicusspecific primer ND4korF (5'-CCCCATTTAACCCCC AATAT-3'), together with the primers N4J8502D(F) and $N 4 \mathrm{~N}-8944 \mathrm{D}(\mathrm{R})$, to amplify a single band of $465 \mathrm{bp}$ in the control species Ae. albopictus and an additional band of $283 \mathrm{bp}$ for Ae. koreicus (Additional file 2: Figure $\mathrm{S} 2$ ). This protocol confirmed the identification as $A e$. koreicus for all individuals previously subjected to morphological observations.

Four other molecular markers were amplified and sequenced: two mitochondrial loci, cytochrome oxidase I (COI) and ND4, and two nuclear (ribosomal) loci, the internal transcribed spacer 2 (ITS2) and the gene portion coding for the $28 \mathrm{~S}$ ribosomal subunit 2 (D2). To amplify these DNA fragments, specific primers and protocols, already published, were followed, as reported in the Additional file 3: Table S1.

The sequences obtained from the PCR products (Eurofins Genomics, Ebersberg, Germany) were used to build phylogenetic trees including sequences from closely related species, retrieved using the nucleotide Basic Local Alignment Search Tool (BLAST) [19]. Alignments were obtained using MUSCLE (MUltiple Sequence Comparison by Log-Expectation) [20], with default parameters for nucleotide sequences. After manual editing, we ran PhyML (phylogenetic maximum likelihood ) [21, 22] with automatic selection [23] of the best phylogenetic model for the data and 1000 bootstraps. For all three genes analyzed in this study, the best model turned out to be $\mathrm{GTR}+\mathrm{G}+\mathrm{I}+\mathrm{F}$, and the corresponding trees are shown in Fig. 2.

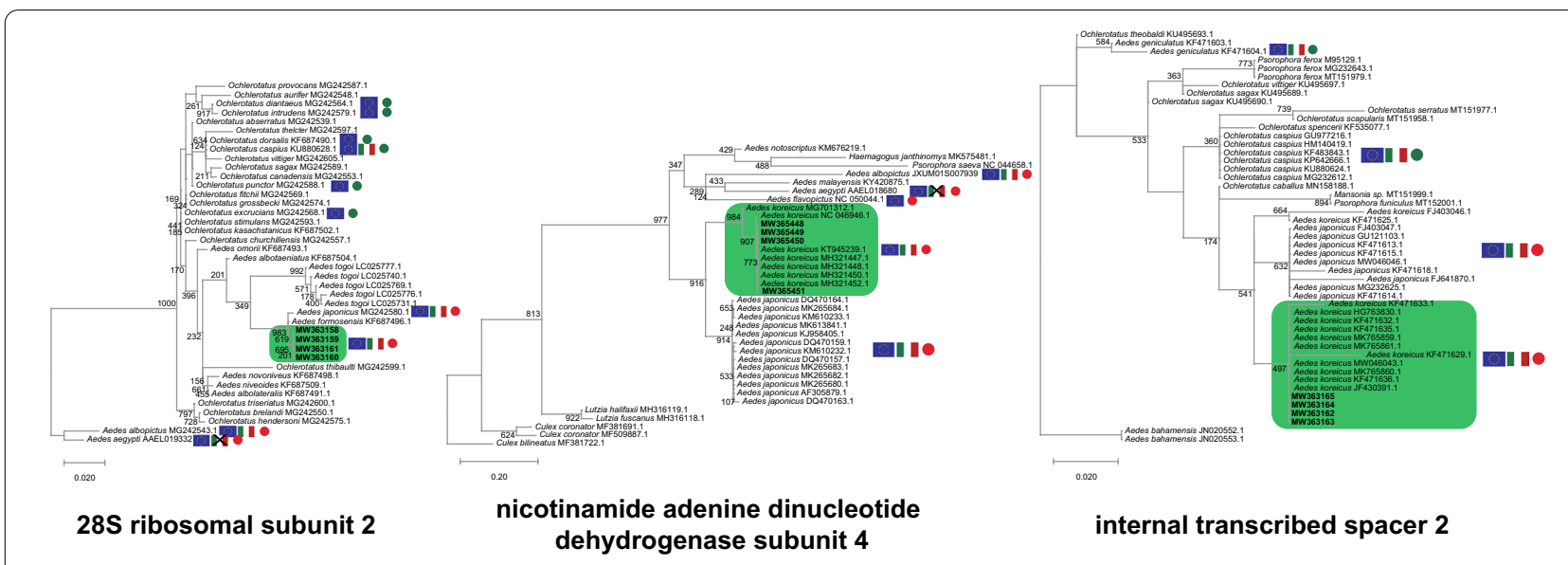

Fig. 2 Phylogenetic trees for D2, ND4, ITS sequence data. Trees were built using PhyML, with automatic model selection, gamma distribution rates (discretized using four categories) and an estimated proportion of invariant sites. Node support was obtained by running 1000 bootstrap replicates. Models selected by SMS (Smart Model Selection) were HKY85 (ND4), K80 (ITS) and GTR (D2). Mosquito species found in Europe, and particularly in Italy, are tagged with the respective national flag. Aedes aegypti, Ae. albopictus, Ae. japonicus and Ae. koreicus are invasive species (red point), while Ochlerotatus diantaeus, Och. intrudens, Och. dorsalis, Och. caspius, Och. punctor and Och. excrucians are native species (green point) 
Because of the lack of enough sequences of related species in GenBank, no tree was constructed for the COI gene. However, we obtained a sequence for Ae. koreicus that showed $98-100 \%$ identity with those in the databases. This sequence and the other three were all deposited in GenBank (MW365452, MW365453, MW365454, MW365455).

The sequences obtained from the amplification of the ND4 region (MW365448, MW365449, MW365450) are $100 \%$ identical to the Ae. koreicus sequences from Germany (GenBank: KT945239.1) and Italy (GenBank: MH321447.1, MH321448.1, MH321450.1, MH321452.1), while only a single nucleotide in the sequence MW365451 was different. As reported in Fig. 2, the ND4 phylogenetic tree highlighted that our sequences clustered with a Korean ND4 sequence (from the mitochondrial genome deposited in GenBank NC 046946.1) with 90.7\% bootstrap support. As expected, the Ae. koreicus monophylum clustered with Ae. japonicus sequences with $91.6 \%$ bootstrap support. In fact, according to the phylogenetic analysis of Cameron et al. [18], Ae. koreicus forms a monophyletic group with the four Ae. japonicus subspecies.

Figure 2 reports also the phylogenetic tree constructed starting from the $D 2$ nuclear region sequences (GenBank: MW363158, MW363159, MW363160, MW363161): our sequences clustered with an Ae. formosensis sequence (GenBank: KF687496.1) and an Ae. japonicus sequence (GenBank: MG242580.1) with 98.3\% bootstrap support. Indeed, the three species are recognized members of the Hulecoeteomyia subgenus [24].

All the four sequences of the ITS2 region (GenBank: MW363162, MW363163, MW363164, MW363165) were identical to Ae. koreicus sequences from Russia, Belgium and Hungary (GenBank: MK765860.1, KF471636.1, JF430391.1, MW046043.1, HG763830.1).

Our results confirm the high genetic similarity between Ae. koreicus, Ae. japonicus and the other species belonging to the Hulecoeteomyia subgenus [4].

All these analyses confirm the presence of Ae. koreicus in the study area. This species is an Asian mosquito species native to Korea, Japan, China and Eastern Russia, which has become increasingly diffused in Europe [11, 12, 16]. Members of this species show great adaptability to temperate regions, probably because Ae. koreicus tolerates low temperatures and prefers mountain regions $\left(23-28^{\circ} \mathrm{C}\right)$ [25]; furthermore, adults of Ae. koreicus have greater persistence during the late summer and autumn seasons [26].

In Italy, Ae. koreicus has colonized an area of more than $3000 \mathrm{~km}^{2}$ in the hills and pre-Alpine zones (from Liguria to Trentino and Veneto regions). The spread of Ae. koreicus mosquitoes is rapidly increasing towards southern and western Italy, from the original infested area in the district of Belluno, probably due to the presence of dense road connections and suitable habitats in other areas [8]. The ability to colonize areas characterized by harsh winter temperatures allows Ae. koreicus mosquitoes to avoid competition for breeding sites occupied by species that have demonstrated a considerable advantage in terms of number and speed of replication, such as Ae. albopictus [26]. In this regard, no specimens of Ae. albopictus were found at the collection site of Ae. koreicus near Bergamo. Larval competition between Ae. albopictus and Ae. koreicus under natural conditions needs to be thoroughly studied and should be integrated with investigations on the interspecific interactions with other species such as Culex spp. and Culiseta spp., found at the same breeding site of Ae. koreicus.

The finding of Ae. koreicus in the pre-Alpine area in the Bergamo district, particularly at the end of the summer season, is a further confirmation of the adaption of these mosquitoes to specific climate and environmental conditions; our georeferenced record will thus contribute to the prediction of future expansion patterns for this species. The distribution area of Ae. koreicus is indeed widening, under the influence of global warming and the heavier international trade and travels in Europe [27]. Additionally, several laboratory experiments suggest that Ae. koreicus is a potential vector of pathogens, such as the nematodes Dirofilaria immitis and Brugia malayi [28] and viruses that cause chikungunya [29] and Japanese encephalitis [30]. The identification of Ae. koreicus during a sampling that was not focused on the collection of Aedes mosquitoes highlights the importance of the monitoring activity and the need for wider knowledge of the introduction routes and the ecological niches occupied by this invasive mosquito. The clarification of some aspects of the biology of the Korean mosquito, such as biting habits, together with a deeper investigation of its vector status, will be fundamental to evaluate the risk of the spread of Ae. koreicus for public health in Europe.

The morphological features of the population of $A e$. koreicus mosquitoes caught in the Bergamo district are comparable to those typical of the Jeju Island population, from which the majority of European populations seem to derive. This observation raises questions about the introduction and expansion of this alien species in Italy and, more broadly, in Europe. One plausible way of introduction in the Alpine area of Lombardy is the exchange of goods and people occurring in the international airport of Orio al Serio, in the Bergamo district. The presence of an international airport was also suggested as the possible importation route to the Liguria region [14]. Alternatively, Ae. koreicus could have been introduced in the Bergamo area from other infested 
areas in Italy. It must be noted that the road transport in this region is very intense, and previous surveys have reported the presence of the species in the nearby districts of Vicenza and Verona, in the Veneto region [8]. Dispersal models predict that parts of the Po and Adige valleys will be colonized by Ae. koreicus in the next decade due to the presence of trade roads [31]. Since control measures to eradicate this species have not yet been applied, an accurate means of monitoring might allow us to reconstruct and follow the dispersal routes of this alien species, and thus to plan targeted interventions aimed at the containment of its spread.

In conclusion, we report the presence of Ae. koreicus in the district of Bergamo, which confirms the rapid spread of this species in this area and raises concerns about its possible role as a vector of diseases in the Alpine area.

\begin{abstract}
Abbreviations
COl: Cytochrome oxidase I; ND4: Nicotinamide adenine dinucleotide dehydrogenase subunit 4; ITS2: Internal transcribed spacer 2; D2: 28 ribosomal subunit 2; PCR: Polymerase chain reaction; SNP: Single-nucleotide polymorphism; BLAST: Basic Local Alignment Search Tool; PhyML: Phylogenetic Maximum Likelihood; SMS: Smart Model Selection.
\end{abstract}

\section{Supplementary Information}

The online version contains supplementary material available at https://doi. org/10.1186/s13071-021-05031-7.

Additional file 1: Figure S1. Localization of the collection site of Aedes koreicus mosquitoes in the Bergamo district (Lombardy Region, Italy) on Google Earth.

Additional file 2: Figure S2. Agarose gel electrophoresis showing results of Aedes koreicus assay with N4J8502D(F), N4N-8944D(R) and ND4korF primers. Ae. albopictus specimen displayed a single fragment of $465 \mathrm{bp}$ (code number 1). Ae. koreicus specimens produced two fragments, one common fragment of $465 \mathrm{bp}$ and the species-specific fragment of $283 \mathrm{bp}$ (code number 3-7). M molecular weight standard (BenchTop 100 bp DNA Ladder, PROMEGA).

Additional file 3: Table S1. Primer sequences and references used for Aedes koreicus identification. PCRs were performed for two mitochondrial loci, cytochrome oxidase I (COI) and nicotinamide adenine dinucleotide dehydrogenase subunit 4 (ND4), and for two nuclear (ribosomal) loci, the internal transcribed spacer 2 (ITS2) and the 285 ribosomal subunit 2 (D2).

\section{Acknowledgements}

The authors are grateful to Norman Bergamelli for his assistance in the field collections and Davide Sassera for the revision of the manuscript.

\section{Authors' contributions}

$A N, I A, S E$, and PG performed sample collections and wrote the first draft of the paper. AN and IA performed molecular analysis and MB analyzed the sequences. PG and SE performed morphological analysis. CB contributed to the design of the study and to the discussion. All authors read and approved the final manuscript.

\section{Funding}

This research was supported by the Italian Ministry of Education, University and Research, PRIN 2017 Prot. 2017J8JR57 to CB and by the Fondazione Cariplo (2017-0798) to PG.

\section{Availability of data and materials}

The nuclear and mitochondrial DNA sequences obtained in this study were deposited in the GenBank database under the accession numbers MW365452-MW365455 for the COl gene, MW365448-MW365451 for the ND4 region, MW363158-MW363161 for the D2 region and MW363162-MW363165 for the ITS2 region.

\section{Declarations}

Ethics approval and consent to participate

Not applicable.

\section{Consent for publication}

Not applicable.

\section{Competing interests}

The authors declare that they have no competing interests.

\section{Author details}

'Department of Biosciences and Pediatric Clinical Research Center "Romeo Ed Enrica Invernizzi", University of Milan, 20133 Milan, Italy. ${ }^{2}$ Department of Biology and Biotechnology, University of Pavia, 27100 Pavia, Italy. ${ }^{3}$ University School of Advanced Studies Pavia, IUSS, 27100 Pavia, Italy. ${ }^{4}$ Italian Malaria Network, Inter University Center for Malaria Research, University of Milan, 20133 Milan, Italy.

Received: 22 March 2021 Accepted: 22 September 2021

Published online: 14 October 2021

\section{References}

1. Semenza JC, Suk JE. Vector-borne diseases and climate change: a European perspective. FEMS Microbiol Lett. 2018;365:fn×244.

2. Cebrián-Camisón S, de la Puente J, Figuerola J. A literature review of host feeding patterns of invasive Aedes mosquitoes in Europe. Insects. 2020;11:848.

3. Edwards FW. Notes on Culicidae with descriptions of new species. Bull of Entomol Res. 1917;7:201-29.

4. Tanaka K, Mizusawa K, Saugstad ES. A revision of the adult and larval mosquitoes of Japan (including the Ryukyu Archipelago and the Ogasawara Islands) and Korea (Diptera: Culicidae). Contrib Am Entomol Inst. 1979;16:1-987.

5. Versteirt V, Pecor JE, Fonseca DM, Coosemans M, van Bortel W. Confirmation of Aedes koreicus (Diptera: Culicidae) in Belgium and description of morphological differences between Korean and Belgian specimens validated by molecular identification. Zootaxa. 2012;3191:21-32.

6. Capelli G, Drago A, Martini S, Montarsi F, Soppelsa M, Delai N, et al. First report in Italy of the exotic mosquito species Aedes (Finlaya) koreicus, a potential vector of arboviruses and filariae. Parasit Vectors. 2011;4:188.

7. Montarsi F, Martini S, Dal Pont M, Delai N, Ferro Milone N, Mazzucato M, et al. Distribution and habitat characterization of the recently introduced invasive mosquito Aedes koreicus [Hulecoeteomyia koreica], a new potential vector and pest in north-eastern Italy. Parasit Vectors. 2013;6:292.

8. Montarsi F, Drago A, Martini S, Calzolari M, De Filippo F, Bianchi A, et al. Current distribution of the invasive mosquito species, Aedes koreicus [Hulecoeteomyia koreica] in northern Italy. Parasit Vectors. 2015;8:614.

9. Baldacchino F, Montarsi F, Arnoldi D, Barategui C, Ferro Milone N, Da Rold G, et al. A 2-yr mosquito survey focusing on Aedes koreicus (Diptera: Culicidae) in northern Italy and implications for adult trapping. J Med Entomol. 2017:54(3):622-30.

10. Gradoni F, Bertola M, Carlin S, Accordi S, Toniolo F, Visentin P, et al. Geographical data on the occurrence and spreading of invasive Aedes mosquito species in Northeast Italy. Data Brief. 2021;36:107047.

11. Suter T, Flacio E, Fariña BF, Engeler L, Tonolla M, Müller P. First report of the invasive mosquito species Aedes koreicus in the Swiss-Italian border region. Parasit Vectors. 2015;8:402.

12. Kalan K, Šušnjar J, Ivović V, Buzan E. First record of Aedes koreicus (Diptera, Culicidae) in Slovenia. Parasitol Res. 2017;116(8):2355-8. 
13. Werner D, Zielke DE, Kampen H. First record of Aedes koreicus (Diptera: Culicidae) in Germany. Parasitol Res. 2016;115(3):1331-4.

14. Ballardini M, Ferretti S, Chiaranz G, Pautasso A, Riina MV, Triglia G, et al. First report of the invasive mosquito Aedes koreicus (Diptera: Culicidae) and of its establishment in Liguria, northwest Italy. Parasit Vectors. 2019;12(1):334.

15. Pfitzner WP, Lehner A, Hoffmann D, Czajka C, Becker N. First record and morphological characterization of an established population of Aedes (Hulecoeteomyia) koreicus (Diptera: Culicidae) in Germany. Parasit Vectors. 2018;11(1):662.

16. Kurucz K, Kiss V, Zana B, Schmieder V, Kepner A, Jakab F, et al. Emergence of Aedes koreicus (Diptera: Culicidae) in an urban area, Hungary, 2016. Parasitol Res. 2016;115(12):4687-9.

17. Fuehrer HP, Schoener E, Weiler S, Barogh BS, Zittra C, Walder G. Monitoring of alien mosquitoes in Western Austria (Tyrol, Austria, 2018). PLoS Negl Trop Dis. 2020;14(6):e0008433.

18. Cameron EC, Wilkerson RC, Mogi M, Miyagi I, Toma T, Kim H-C, et al. Molecular phylogenetics of Aedes japonicus, a disease vector that recently invaded Western Europe, North America, and the Hawaiian Islands. J Med Entomol. 2010;47(4):527-35.

19. Nucleotide Basic Local Alignment Search Tool. https://www.ncbi.nIm.nih. gov/gene/. Accessed 4 Dec 2020.

20. Muscle. https://www.ebi.ac.uk/Tools/msa/muscle/. Accessed 4 Dec 2020.

21. Phylogenetic Maximum Likelihood. http://www.atgc-montpellier.fr/ phyml/. Accessed 5 Dec 2020

22. Guindon S, Dufayard J-F, Lefort V, Anisimova M, Hordijk W, Gascuel O. New algorithms and methods to estimate maximum-likelihood phylogenies: assessing the performance of PhyML 3.0. Syst Biol. 2010;59(3):307-21.

23. Lefort V, Longueville J-E, Gascuel O. SMS: Smart Model Selection in PhyML. Mol Biol Evol. 2017;34(9):2422-4.

24. Somboon P, Phanitchakun T, Namgay R, Harbach RE. Description of Aedes (Hulecoeteomyia) bhutanensis n. sp. (Diptera: Culicidae) from Bhutan. Acta Trop. 2020;203:105280.
25. Marini G, Arnoldi D, Baldacchino F, Capelli G, Guzzetta G, Merler S, et al. First report of the influence of temperature on the bionomics and population dynamics of Aedes koreicus, a new invasive alien species in Europe. Parasit Vectors. 2019;12(1):524.

26. Baldacchino F, Arnoldi D, Lapère C, Rosà R, Montarsi F, Capelli G, et al. Weak larval competition between two invasive mosquitoes Aedes koreicus and Aedes albopictus (Diptera: Culicidae). J Med Entomol. 2017:54(5):1266-72.

27. Roiz D, Wilson AL, Scott TW, Fonseca DM, Jourdain F, Müller P, et al. Integrated Aedes management for the control of Aedes-borne diseases. PLOS Negl Trop Dis. 2018;12(12):e0006845.

28. Montarsi F, Ciocchetta S, Devine G, Ravagnan S, Mutinelli F, di Regalbono A, et al. Development of Dirofilaria immitis within the mosquito Aedes (Finlaya) koreicus, a new invasive species for Europe. Parasit Vectors. 2015;8:177.

29. Ciocchetta S, Prow NA, Darbro JM, Frentiu FD, Savino S, Montarsi F, et al. The new European invader Aedes (Finlaya) koreicus: a potential vector of Chikungunya virus. Pathog Glob Health. 2018;112(3):107-14.

30. Miles JAR. Some ecological aspects of the problem of arthropod-borne animal viruses in the Western Pacific and South-East Asia regions. Bull World Health Organ. 1964;30(2):197-210.

31. Marcantonio M, Metz M, Baldacchino F, Arnoldi D, Montarsi F, Capelli G, et al. First assessment of potential distribution and dispersal capacity of the emerging invasive mosquito Aedes koreicus in Northeast Italy. Parasit Vectors. 2016;9:63.

\section{Publisher's Note}

Springer Nature remains neutral with regard to jurisdictional claims in published maps and institutional affiliations.
Ready to submit your research? Choose BMC and benefit from:

- fast, convenient online submission

- thorough peer review by experienced researchers in your field

- rapid publication on acceptance

- support for research data, including large and complex data types

- gold Open Access which fosters wider collaboration and increased citations

- maximum visibility for your research: over $100 \mathrm{M}$ website views per year

At BMC, research is always in progress.

Learn more biomedcentral.com/submissions 